\title{
On the Coexistence of UWB with Fixed Wireless Access Systems
}

\author{
Romeo Giuliano, Gianluca Guidoni, Franco Mazzenga, and Francesco Vatalaro \\ University of Rome - Tor Vergata and RADIOLABS \\ Viale del Politecnico 1, 00133 Rome - ITALY \\ \{giuliano, guidoni, mazzenga, vatalaro\}@radiolabs . it
}

\begin{abstract}
Ultra Wide Bandwidth (UWB) spread-spectrum techniques are getting an increased importance due to their many desirable features such as high bit rates support and low power consumption. UWB is considered one enabling technology for advanced wireless local area networks providing integrated multimedia services to nomadic users over hot-spot areas. The assessment of the interference caused by UWB devices on already existing systems is fundamental to guarantee not conflicting coexistence and, therefore, the acceptance of this new technology worldwide. In this paper we use simulation to analyze the coexistence issues between and indoor UWB system and an outdoor fixed wireless access (FWA) system in the $3.5 \div 5.0 \mathrm{GHz}$ frequency range. We consider a realistic UWB system architecture and we show that in many cases UWB system can coexist with FWA without causing dangerous interference.
\end{abstract}

\section{Introduction}

Ultra Wide Bandwidth (UWB) spread-spectrum techniques are gaining increased importance due to their many desirable features such as supported high bit rates and low power consumption [1]. In order to protect existing services against UWB interference the Federal Communications Commission (FCC) has restricted the UWB operating frequencies and allowed UWB transmissions under the operating conditions stated by frequency-power masks issued for each UWB application/device. As a result, UWB communications are confined to the frequency range of $3.1 \div 10.6 \mathrm{GHz}$.

The UWB technology is based on the transmission of very short duration pulses and the information signal is spread over a very wide bandwidth (of the order of several $\mathrm{GHz}$ ) 2. UWB is one viable candidate for short-range indoor radio communications supporting very high bit rates services and applications. It enables the implementation of innovative wireless local area networks providing multimedia services to nomadic users over hot-spot areas. Thus the assessment of the possible interference caused by UWB devices is of fundamental importance to guarantee not conflicting coexistence between UWB devices and any other

\footnotetext{
* Work done for RADIOLABS, Consorzio Università Industria - Laboratori di Radiocomunicazioni, Italy within the IST ULTRAWAVES European Project
} 
system and to obtain acceptance of this new technology worldwide. To meet the UWB interference requirements on the existing narrow-band systems UWB low transmission-power and short-range operations have to be ensured. The ITU document [3] contains a preliminary analysis on the possible interference of indoor UWB terminals onto an outdoor Fixed Wireless Access (FWA) system. However results in [3] are based on assumptions leading to pessimistic conclusions about the impact of UWB on FWA systems. Main misleading assumptions deal with the selection of the free space channel model for both indoor and outdoor propagation and to the procedure used to evaluate UWB interference on the FWA receiver. In particular in 3] it is assumed that each UWB terminal continuously transmits at its maximum power without accounting for (possible) power control. In this paper we analyze the coexistence problems between indoor UWB system and an outdoor FWA system through computer simulation. In order to compare our results with those already presented in the current literature we keep many of the original assumptions in [3]. However we account for additional UWB system features such as: the UWB system architecture, more realistic propagation models, UWB terminal activity factor. We demonstrate that in many practical cases UWB system can coexist with FWA without dangerous interference. The paper is organized as follows. In Section 2 we summarize the main characteristics of the considered FWA systems and we assess the FWA interference requirements. In Section 3 we describe the selected scenarios and we define the UWB system features introducing the main parameters describing its usage modes. In Section 4 we define the assessment methodology introduced in this work. Simulation results presented and discussed in Section 5. Finally conclusions are given in Section 6 .

\section{Interference Limits Requirements}

To assess the limits of UWB interference on the existing FWA systems we start from the ITU-R requirements in 4] concerning the interference from unwanted emissions from sources other than fixed service (FS) or services sharing the same band on primary bases. As indicated in [4] the total interference to a fixed service link is partitioned as:

- $89 \%$ for the intra service interference;

- $10 \%$ for the co-primary services interference;

$-1 \%$ for the aggregation of the following interference sources:

- Emissions from radio services which share frequency allocations on a non-primary basis;

- Unwanted emissions (i.e. out-of-band and spurious emissions such as energy spread from radio systems, etc.) in non-shared bands;

- Unwanted radiations (e.g. ISM applications);

As indicated in [5] and [6] the previous percentages apply to the performance objectives. In addition, this degradation allowance refers to the aggregation of the whole secondary services transmitters and unwanted signals. UWB systems 
are commonly classified as secondary services therefore this criterion is applied. The ITU-R has defined the following long and short term interference criteria for not conflicting coexistence.

\section{Long term criteria (20\% of the time)}

- For co-primary sharing a margin degradation of $0.5 \mathrm{~dB}$, equivalent to a $I / N=-10 \mathrm{~dB}$, leads to a performance degradation of $10 \%$.

- For secondary service interference and unwanted emissions, the performance degradation shall not exceed $1 \%$. For these services it was concluded that a $I / N=-20 \mathrm{~dB}$ is the adequate criteria since it represents a margin degradation of $0.04 \mathrm{~dB}$.

The previous two statements indicate generic objectives and implicitly assume that interference has spectral characteristic similar to white noise. However in the UWB case, due to the pulsed characteristic of UWB signals separate considerations would be needed for both average (RMS) and peak interference objectives within the FS receiver bandwidth. It should be observed that the allocation of $99 \%$ of the interference margin to intra-service and/or co-primary services $(89 \%+10 \%)$ might also be too pessimistic with today's technology and in more realistic conditions higher margins than 1\%, in particular $I / N$ much closer to $0 \mathrm{~dB}$, could be tolerated by fixed links for secondary services. In the rest of the paper we mention the limits and results both considering the longterm interference with the conservative $I / N=-20 \mathrm{~dB}$ and with $I / N=-10$ dB.

Short term criteria (0.0001\% of time)

Short-term criteria in 7 gives allowance for a positive (in dB) $I / N$ ratio to happen for very short percentage of time. Positive $I / N$ ratio can be related to peak interference. Similarly to [3] this criterion will not be considered in this paper.

The FWA system considered in this paper includes two communicating devices: a FWA Central station (CS) positioned on the roof of the building, and a FWA Terminal Station (TS) close to the building. The most relevant characteristic to our interference study is the FWA receiver noise parameter. This can be expressed as:

$$
N_{A}=-144+10 \log _{10}\left(R X_{B W}\right)+N F
$$

where $R X_{B W}$ is the $3 \mathrm{~dB}$ receiver bandwidth (in $\mathrm{MHz}$ ) and $N F$ is the receiver noise figure $(\mathrm{dB})$.

Considering a FWA receiver operating at $3.5 \mathrm{GHz}$, we assume $N F=5 \mathrm{~dB}$, and using (1) we obtain $N_{A 50}=-122 d B W$ for $R X_{B W}=50 \mathrm{MHz}$ and $N_{A 14}=$ $-127.5 \mathrm{dBW}$ for $R X_{B W}=14 \mathrm{MHz}$. Considering FWA systems operating in the $3.6 \div 4.2 \mathrm{GHz}$ and $4.4 \div 5.0 \mathrm{GHz}$ we assume $N F=6 \mathrm{~dB}$ and for $R X_{B W}=50 \mathrm{MHz}$ we obtain $N_{A 50}=-121 \mathrm{dBW}$.

Starting from the calculated FWA receiver noise characteristics, we obtained the limits on the allowed UWB inter-system interference indicated in Table [1] 
Table 1. Summary of UWB aggregate interference objectives for coexistence with FS-ITU-R objectives

\begin{tabular}{ll}
\hline FWA features & Interference limits \\
\hline FWA CS $\left(B_{W}=14 \mathrm{MHz}\right)$ & $\leq-159 \mathrm{dBW} / \mathrm{MHz} @ \mathrm{I} / \mathrm{N}=-20 \mathrm{~dB}$ \\
& $\leq-149 \mathrm{dBW} / \mathrm{MHz} @ \mathrm{I} / \mathrm{N}=-10 \mathrm{~dB}$ \\
FWA CS $\left(B_{W}=50 \mathrm{MHz}\right)$ & $\leq-159 \mathrm{dBW} / \mathrm{MHz} @ \mathrm{I} / \mathrm{N}=-20 \mathrm{~dB}$ \\
& $\leq-149 \mathrm{dBW} / \mathrm{MHz} @ \mathrm{I} / \mathrm{N}=-10 \mathrm{~dB}$ \\
\hline
\end{tabular}

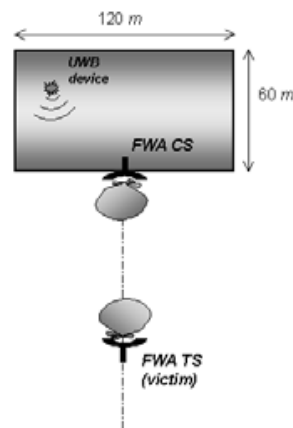

(a) - building upper view

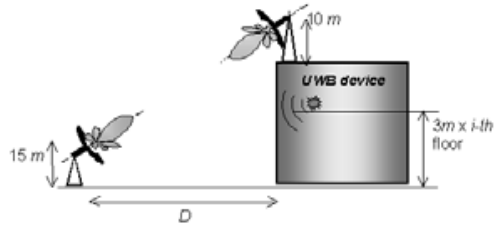

(b) - building side view

Fig. 1. Hot spot reference scenario for FWA - UWB system coexistence.

Also the corresponding values for the less conservative, but still very safe, protection levels $I / N=-10 \mathrm{~dB}$ were included. To study the effects of UWB on the FWA we first evaluate the power of the UWB interference at the FWA receiver $I_{U W B \rightarrow F W A}$. The power spectral density of the UWB interference is obtained by dividing $I_{U W B \rightarrow F W A}$ for the bandwidth of the UWB signal. The result is then compared with the values in Table 1 .

The interference $I_{U W B \rightarrow F W A}$ is evaluated as:

$$
I_{U W B \rightarrow F W A}=\sum_{n} P_{T_{n}} L\left(d_{n}\right),
$$

where $P_{T_{n}}$ is the power transmitted by the $n$-th UWB terminal and $L\left(d_{n}\right)$ is the path loss between the $n$-th UWB terminal and the FWA receiver located at relative distance $d_{n}$. The path loss $L(d)$ accounts for both indoor and outdoor propagation and antenna gains.

\section{$3 \quad$ Selected Scenario}

To evaluate (21) we consider an UWB system located in a commercial/industrial building (hot-spot scenario). We assume that UWB system interferes with the 
Table 2. Main parameters of the considered UWB system

\begin{tabular}{ll}
\hline Simulator feature & Alternatives \\
\hline Outdoor Propagation Model & Free Space, ITU-R P.1411-1 \\
& Cost231-Hata model/3GPP \\
Indoor Propagation Model & Free space, ITU-R P.1238-2, \\
& Cassioli, Molish Win, (CMW) [8 \\
Power Control & Used for every UWB device \\
Activity Factor & $1 \%, 2 \%, 5 \%, 10 \%$ \\
Bit Rates & $2,25,110 \mathrm{Mb} / \mathrm{s}$ \\
\hline
\end{tabular}

FWA system in the proximity of the considered building. The FWA-TS antenna is steered in order to optimally point to the FWA-CS. We assume the building has 10 isolated floors and we neglect the UWB intra-floor interference. The reference scenario is plotted in Figure 1. The UWB system contains a mixed population of devices characterized by different bit rates. UWB devices are located inside building and communicate among them according to a master-slave architecture. To evaluate UWB interference on FWA we introduced more accurate models for indoor and outdoor propagation, power control, terminals activity factors less than unity. The antenna radiation patterns and other geometrical parameters such as the FWA distance from the building, floor dimensions were assumed as in [3]. The parameters and models used for analysis are summarized in Table2, The number of possible scenarios obtained from the combinations of the parameters in Table 2 and that should be explored is very large. However many scenarios could result as unrealistic. As an example it could be noted that using $100 \%$ activity factors contrasts with the typical deployment scenarios envisaged for UWB devices where they will be active only for a fraction of time. In particular it is estimated that in any case the aggregate percentage of time that any single device transmits is to be considered not exceeding $10 \%$ of the time. For brevity, in the following we restrict our analysis to some important and realistic cases.

\section{Interference Calculation}

To evaluate $I_{U W B \rightarrow F W A}$ in (2) we used the software simulator developed within the IST ULTRAWAVES European project [9. We consider an UWB system based on a Code Division Multiple Access (CDMA) technique with multiple parallel radio paths for upstream (slave to master) and downstream (master to slave) communications. The simulated area is assumed to be rectangular and UWB masters are located on a regular grid. Slaves can be arbitrarily and uniformly located within each floor according to a uniform distribution. The simulator is snapshot based and its operating principle is illustrated in the flowchart of Fig.2 In each outer loop iteration a new random scenario is generated. Assuming the positions of the masters scenario generation consists in randomly placing the slaves in the service area. The inner loop is used to simulate the power control procedure. For each inner iteration the power of each transmitter is increased or 


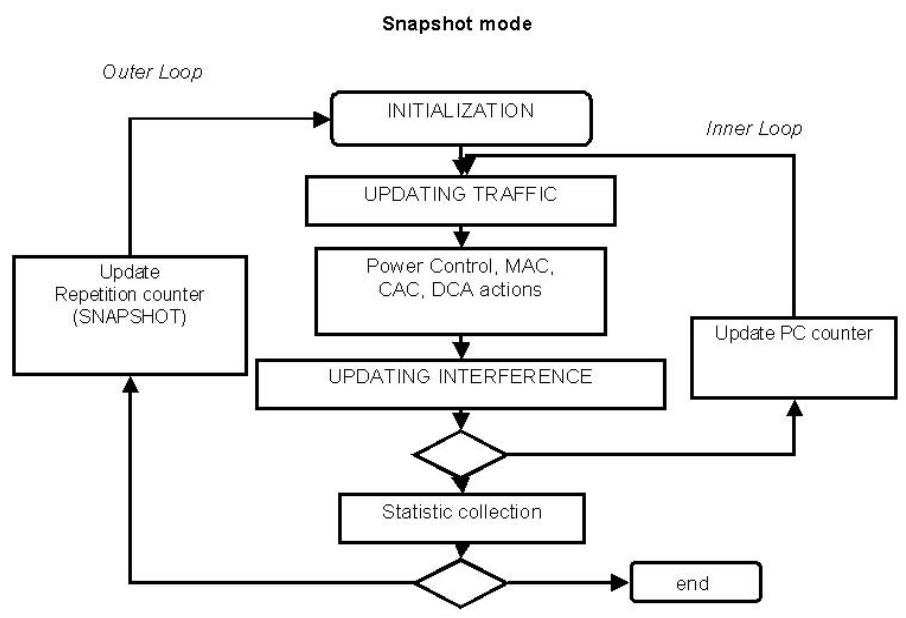

Fig. 2. Simulator flowchart

decreased in accordance to the comparison of the carrier-to-interference ratio, $C / I$, calculated for each slave by the master device to which the slave is connected to, and the reference carrier-to-interference ratio, $(C / I)_{\text {Target }}$. The interference $I$ measured for each master receiver is calculated in each inner iteration accounting for the active UWB transmitters in the area and the propagation losses. The inner procedure is halted when the power transmitted by each slave remains constant or has reached the maximum. After reaching this steady condition all the relevant parameters such as the power level transmitted by each user, the users in outage etc., are collected. More than 10000 snapshots were generated and the statistical behavior of the collected parameters was analyzed. The mobility of the UWB devices was not considered in this paper.

The simulator parameters are now discussed in detail.

\section{- Source Traffic Model}

We consider traffic sources characterized by different bit rates and activity factors. The simulation results presented in the next Section will consider different combinations of source traffic models.

- Transmitted Power and Interference

The maximum transmitting power for each UWB terminal is $-6.2 \mathrm{dBm}$ and can be easily calculated starting from the maximum allowable power spectral density (i.e. $-41 \mathrm{dBm} / \mathrm{MHz}$ ), assuming a UWB signal bandwidth $\left(W_{U W B}\right)$ of $3 \mathrm{GHz}$ and a flat UWB spectrum. The total interference $I$ in each master receiver is:

$$
I=I_{\text {Intra }}+I_{F W A \rightarrow U W B}+\eta
$$

where $I_{\text {Intra }}$ is the intra-system interference caused by the UWB devices; $\eta$ is the thermal noise power and $I_{F W A \rightarrow U W B}$ is the inter-system interference 
due to FWA on the UWB system. The $I_{F W A \rightarrow U W B}$ is accounted for by increasing the noise figure in the UWB receiver by $5 \mathrm{~dB}$. The $(C / I)_{\text {Target }}$ used in the power control procedure for each UWB source was evaluated as

$$
\left(\frac{C}{I}\right)_{\text {Target }}=\left(\frac{E_{b}}{N_{0}}\right)_{\text {Target }}-P G
$$

where $P G=W_{U W B} / R_{b}$ and $R_{b}$ is the source bit rate. The UWB reference $\left(E_{b} / N_{0}\right)_{\text {Target }}$ was always set to $4 \mathrm{~dB}$ in each scenario and for each bit rate.

- Pathloss models: indoor propagation

To analyze the dependence of the UWB interference from the selected channel model, we repeated the simulations assuming two different path-loss models: the simple free space model and the Cassioli-Molish-Win (CMW) model 8] repeated below:

- Free space propagation model

$$
A_{F S}(d)=c_{0}+c_{1} \log _{10}(d) \quad[d B]
$$

where $c_{0}=$ pathloss at $1 \mathrm{~m}=20 \cdot \log _{10}\left(\frac{4 \pi}{\lambda}\right)=43.32 \mathrm{~dB}$ at $3.5 \mathrm{GHz} ; c_{1}=20$ $\mathrm{dB} / \mathrm{m}$

- $C M W$ model

$$
A_{C M W}(d)= \begin{cases}0 & d \in[0,1] \\ c_{0} \cdot \log _{10}(d) & d \in\left[1, d_{\text {break }}\right] \\ c_{1}+c_{2} \cdot \log _{10}\left(\frac{d}{d_{\text {break }}}\right) & d>d_{\text {break }}\end{cases}
$$

it is a dual slope model where $c_{0}=20.4 \mathrm{~dB} / \mathrm{m}, c_{1}=20.4 \log _{10}\left(d_{\text {break }}\right)$, $c_{2}=74 \mathrm{~dB} / \mathrm{m}$ and $d_{\text {break }}=11 \mathrm{~m}$.

The selected path loss models represent two extremes among those indicated in Table 1. In fact, neglecting possible tunnel effects, the path loss exponents of the two selected models vary between 2 and 7.4 thus accounting for many intermediate values of the path loss exponents of other models.

- Antenna models

We assumed that any UWB device has an omnidirectional antenna on the horizontal plane with gain 0dBi. For the FWA-TS, we use the antenna model indicated in [10. It is a sectorial antenna with sectors of $90^{\circ}$ and a main lobe gain of $16 \mathrm{dBi}$. Its radiation pattern envelopes are reported in [10].

Call admission control strategies (CAC) strategies enhance performance and allow to reduce the interference both in the UWB system and other narrowband systems. For example an UWB slave could be stopped from transmitting when its power level requirements are judged too high by the master. The beneficial effects of CAC strategies were not considered in this paper. 


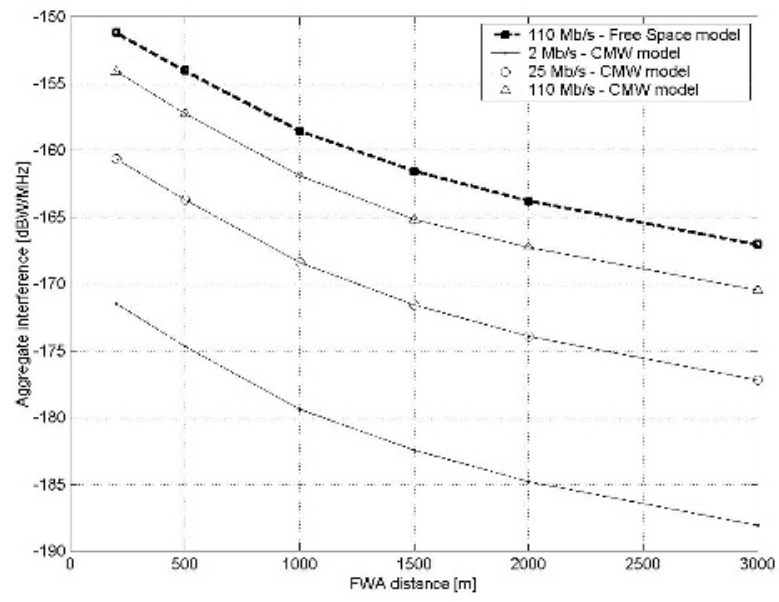

Fig. 3. UWB aggregate interference vs FWA distance - Single Service Case - Free Space and CMW channel models - 32 masters per floor, activity factor 1

\section{$5 \quad$ Simulations Results}

Simulations results were obtained considering different combinations of the system parameters indicated in Table 2. In this paper only upstream (slave-tomaster) transmissions were considered. To validate our simulator we first reimplemented the scenario in 3] and we considered an UWB terminal density of 450 users per floor. The floor dimensions where $120 \times 60 \mathrm{~m}^{2}$ ) and the vertical distance between floors is $3 \mathrm{~m}$ (see Fig 1). Every UWB device is unrealistically set to transmit $100 \%$ of the time at its maximum power level (i.e. $-6.2 \mathrm{dBm}$ for $W_{U W B}=3 \mathrm{GHz}$ ). The UWB devices were positioned over a grid at a relative distance of $4 \mathrm{~m}$. We used the free space path loss model for both indoor and outdoor links. The results obtained are in very good agreement with those presented in 3. and for brevity are not reported. In the following subsections we illustrate the simulation results in more realistic scenarios considering both single and multiple bit rate services.

\subsection{Single Service Cases}

We assume that UWB devices transmit at the same bit rate: 2, 25 or $110 \mathrm{Mb} / \mathrm{s}$. The density of the UWB terminals is varied and both free space and CMW models have been considered. The building penetration loss is neglected 1 .

${ }^{1}$ For a correct evaluation of interference at least $10 \mathrm{~dB}$ should be considered bringing the target aggregate interference to $-139 \mathrm{dBW} / \mathrm{MHz} @ I / N=-10 \mathrm{~dB}$ (or -149 $\mathrm{dBW} / \mathrm{MHz} @ I / N=-20 \mathrm{~dB})$ 


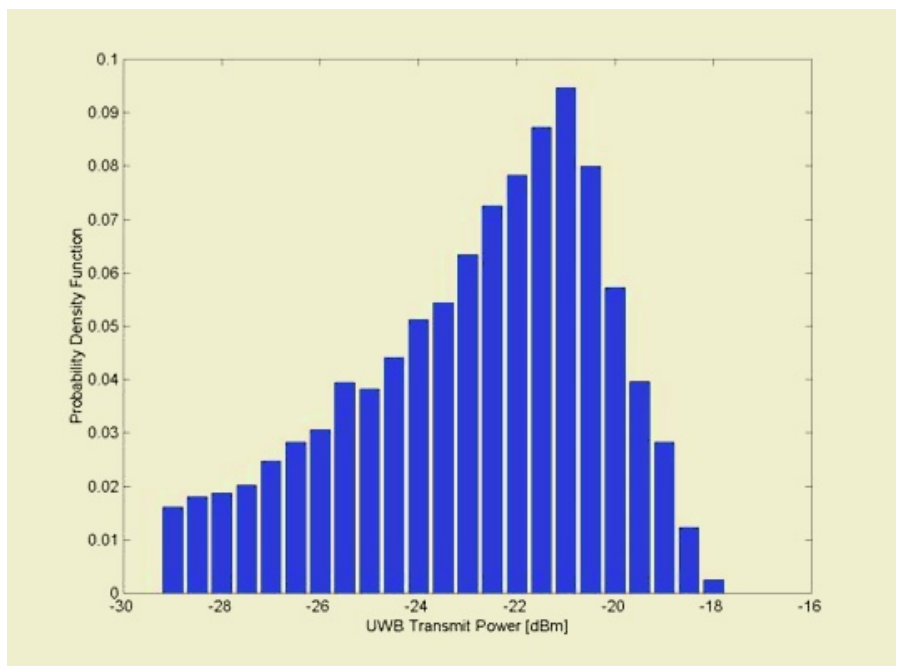

Fig. 4. Power distribution for (power controlled) devices transmitting at $110 \mathrm{Mb} / \mathrm{s}$

In Fig. 3] we plot the UWB aggregate interference density vs the distance of the FWA-TS from the building. We considered the free space and the CMW channel models for indoor propagation while we always adopted the free space channel model for outdoor. A density of 500 UWB devices per floor was considered. From the results in Fig 3 it can be noticed that due to power control action the aggregate interference $(\mathrm{dBW} / \mathrm{MHz})$ is lower than the ITU requirements in Table 1 in any conditions and for practical FWA distances from the building. Including $10 \mathrm{~dB}$ due to building penetration loss, the target aggregate interference is fully respected, even at these extreme high densities.

In Fig. 4 we plot the histogram of the power transmitted by slaves operating at $110 \mathrm{Mb} / \mathrm{s}$. It can be observed that no UWB users are in outage and that due to power control action the transmitted power is always well below than the FCC limit of $-6.2 \mathrm{dBm}$.

Having proved the compatibility between the UWB and the FWA systems with different service classes and for densities higher than those indicated in [3], we now vary the activity factor to the maximum levels foreseeable for an UWB terminal from $1 \%$ up to $10 \%$.

In Fig 5 and Fig 6 we plot the aggregate interference density for different FWA receiver distance with transmitters at $110 \mathrm{Mb} / \mathrm{s}$ and for different activity factors. As expected, results in Fig[5 and Fig [6 show the raise in the aggregate interference levels with the increase of activity from $1 \%$ to $5 \%$ and then to the maximum expected of 10\%. Always from the results in Fig 5 and Fig [6 even in the unrealistic case of 500 UWB devices per floor and even increasing the activity factor to $10 \%$, in most practical cases the effects on the FWA system are not harmful. 


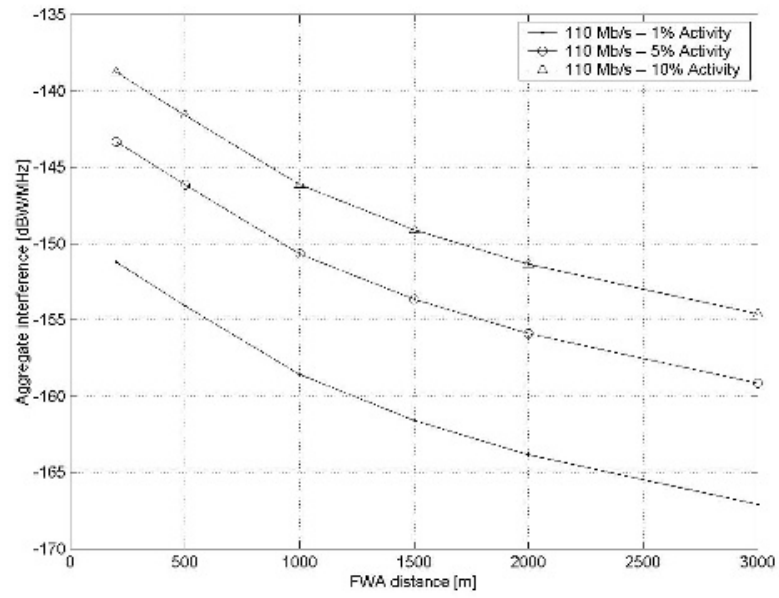

Fig. 5. Aggregate interference density for different FWA receiver distance with transmitters at $110 \mathrm{Mb} / \mathrm{s}$, free space indoor model, Activity factors of 1\%, 5\% and $10 \%$

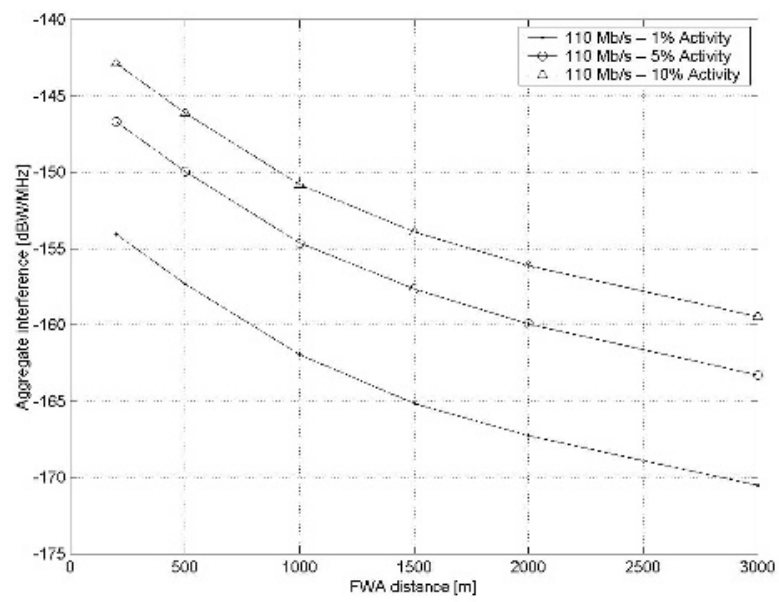

Fig. 6. Aggregate interference density for different FWA receiver distance with transmitters at $110 \mathrm{Mb} / \mathrm{s}, \mathrm{CMW}$ indoor model, Activity factors of 1\%, 5\% and $10 \%$

\subsection{Multi-service Cases}

In this Section we consider a scenario where UWB terminals simultaneously operate at different bit rates. The first set of simulations considered slaves with two different bit rates: $2 \mathrm{Mb} / \mathrm{s}$ ( $50 \%$ of slaves) and $110 \mathrm{Mb} / \mathrm{s}$ (50\% of slaves - possibly unrealistic). The system parameters are unchanged but the devices 


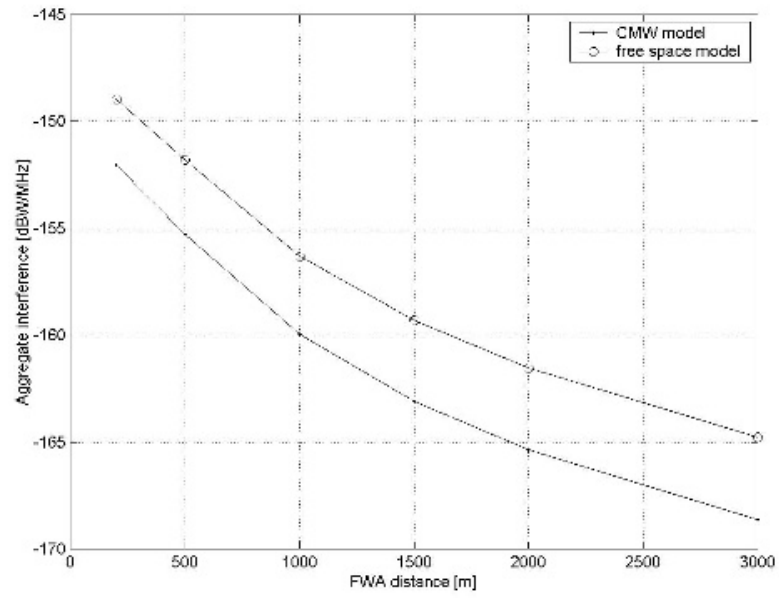

Fig. 7. Aggregate interference density vs FWA receiver distance - UWB services 110 $\mathrm{Mb} / \mathrm{s}(50 \%)+2 \mathrm{Mbit} / \mathrm{s}(50 \%)$

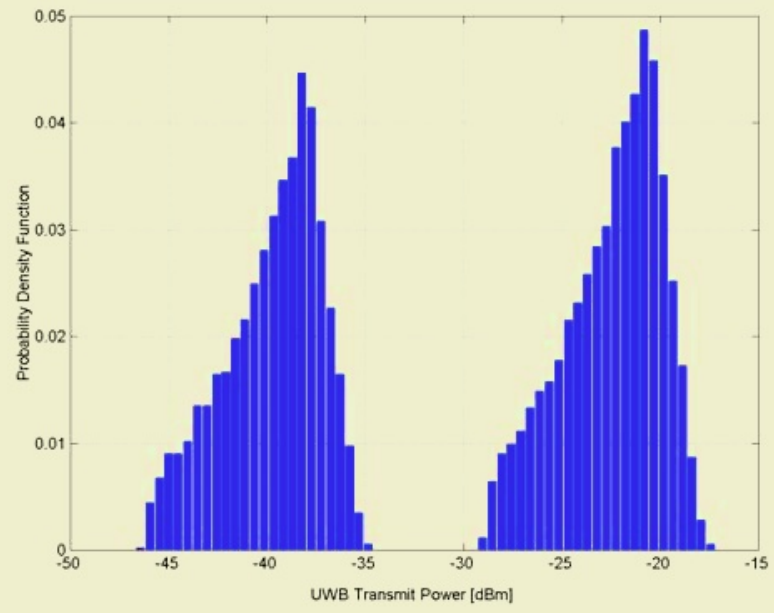

Fig. 8. Power distribution for power controlled devices transmitting at $110 \mathrm{Mb} / \mathrm{s}$ and $2 \mathrm{Mbit} / \mathrm{s}$ (50\%)- indoor free space channel model

density is reduced to 300 UWB terminals per floor to obtain a more realistic scenario. Slaves are power controlled and have an activity factor of $5 \%$.

In Fig[7 we plot the aggregate interference density vs the FWA receiver distance from the building. Different indoor propagation models were considered. From the results in Fig:7 it can be observed that full compatibility between 


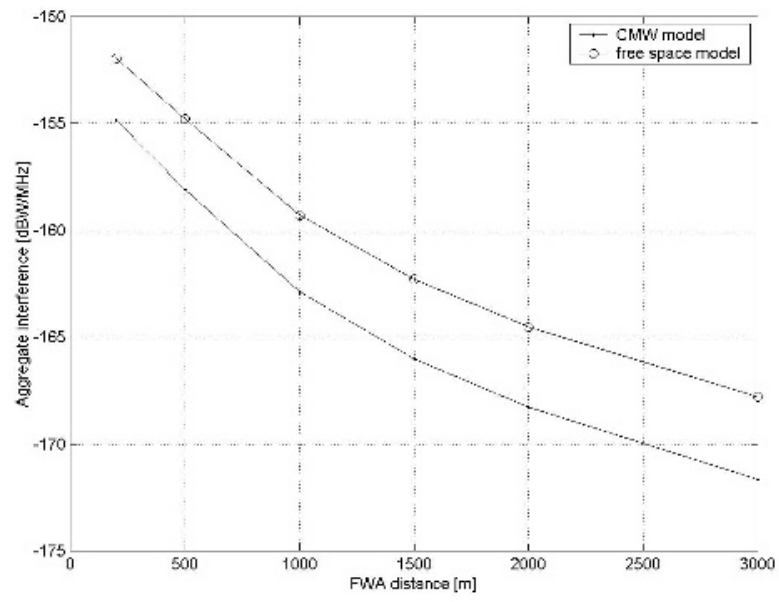

Fig. 9. Aggregate interference density vs FWA receiver distance - UWB services $110+\mathrm{Mb} / \mathrm{s}(20 \%)+25 \mathrm{Mb} / \mathrm{s}(30 \%)+2 \mathrm{Mbit} / \mathrm{s}(50 \%)$-Power control ON - free space and CMW indoor propagation model

Table 3. Traffic Parameters for mixed bit rate simulation: $2+25+110 \mathrm{Mb} / \mathrm{s}$.

\begin{tabular}{ccc}
\hline Population [\% of the total] & Activity Factor & Data Rate \\
\hline 20 & $2 \%$ & $110 \mathrm{Mb} / \mathrm{s}$ \\
30 & $5 \%$ & $25 \mathrm{Mb} / \mathrm{s}$ \\
50 & $10 \%$ & $2 \mathrm{Mb} / \mathrm{s}$ \\
\hline
\end{tabular}

UWB and FWA services is ensured both for free space and CMW models. In fact, for practical FWA distances the aggregate interference is well below the targets in Table 1 that however are met even at very close FWA distances.

In Fig. 8 we plot the histogram on the UWB transmitted power in the case of terminals operating at $2 \mathrm{Mb} / \mathrm{s}$ and $110 \mathrm{Mb} / \mathrm{s}$.

Thanks to power control action the transmitted power is always well below than the FCC limit of $-6.2 \mathrm{dBm}$. As expected the average power transmitted by the $110 \mathrm{Mb} / \mathrm{s}$ terminals is larger than the $2 \mathrm{Mb} / \mathrm{s}$. This is due to the lower coding gain available for the higher bit rate services.

Finally in Fig 9 we plot the aggregate interference by considering a UWB mixed bit rate scenario as a function of the FWA receiver distance. We considered 300 UWB users per floor and 32 UWB master devices. Traffic parameters used to obtain the results in Fig 9 are indicated in Table 3. From the results in Fig.9 it can be observed that full compatibility between UWB and FWA services is ensured both for free space and CMW models. 


\section{Conclusions}

The assessment of the potential interference caused by UWB devices is a fundamental research topic to ensure not conflicting coexistence between UWB devices and any other existing system. Any study needs to go beyond simplistic models and should consider realistic scenarios. As many other wireless local area network systems, UWB terminals will be in sleep mode for the large majority of times, will not run continuously and will not emit constantly at the maximum allowed power. More accurate path loss models were considered to evaluate the UWB aggregate interference on FWA. The results presented in this paper show that considering basic system features such as the UWB system architecture, the activity factor, the power control and including realistic propagation models, even in the extreme densities proposed there is no risk for the FWA operations. Further margins not even considered in this paper still exist such as admission control techniques, antenna polarization mismatches, deep NLOS and multiple trough-wall indoor losses. The analysis procedure introduced in this paper can be easily extended to other significative scenarios.

\section{References}

1. R. A. Scholtz, R. Weaver, E. Homier, J. Lee, P. Hilmes, A. Taha, and R. Wilson, "UWB radio deployment challenges", in Proc. IEEE Int. Symp. on Personal, Indoor and Mobile Radio Communications, vol. 1, pp. 620-625, Sept. 2000. London, UK.

2. M. Ho, L. Taylor, and G. Aiello, "UWB technology for wireless video networking," in Int. Conference on Consumer Electronics, ICCE, pp. 18-19, 2001.

3. ITU-R M18-20A5-1R0-SE24-UWB - annex5-1- FS- Updated UWB and FS coexistence report (Annex 5-1), updated version of: "Preliminary Study on Coexistence between UWB and the Fixed Service in Bands from 1 to $6 \mathrm{GHz}$ "

4. ITU-R F.1094-1 (10/95) "Maximum allowable error performance and availability degradations to digital radio-relay systems arising from interference from emissions and radiations from other sources"

5. ITU-R F.1397: "Error performance objectives for real digital radio links used in the international portion of a $27500 \mathrm{~km}$ hypothetical reference path at or above the primary rate"

6. ITU-R F.1491: "Error performance objectives for real digital radio links used in the national portion of a $27500 \mathrm{~km}$ hypothetical reference path at or above the primary rate"

7. ITU-R F.758-2 (05/00) "Considerations in the development of criteria for sharing between the terrestrial fixed service and other services"

8. D. Cassioli, Moe Z. Win and Andreas F. Molisch, "The ultra-wide bandwidth indoor channel: from statistical model to simulations", IEEE Journal on Selected Areas on Commun. - Special issue "Channel and propagation models for wireless system design," Aug. 2002

9. "UlTRA Wideband Audio Video Entertainment System (ULTRAWAVES)" European Project - IST-2001-35189

10. ITU-R F.1336: "Reference radiation patterns of omnidirectional, sectoral and other antennas in point-to-multipoint systems for use in sharing studies in the frequency range from 1 to about $70 \mathrm{GHz}$ " 